\title{
Shape Representation via Conformal Mapping
}

\author{
Matt Feiszli and David Mumford \\ Division of Applied Mathematics, Brown University, Providence, RI USA 02912
}

\begin{abstract}
Representation and comparison of shapes is a problem with many applications in computer vision and imaging, including object recognition and medical diagnosis. We will discuss some constructions from the theory of conformal mapping which provide ways to represent and compare planar shapes. It is a remarkable fact that conformal maps from the unit disk to a planar domain encode the geometry of the domain in useful and tangible ways. Two examples of the relationship between conformal mapping and geometry are provided by the medial axis and the boundary curvature of a planar domain. Both the medial axis and the boundary curvature can be used in applications to compare and describe shapes and both appear clearly in the conformal structure. Here we introduce some results demonstrating how conformal mapping encodes the geometry of a planar domain.
\end{abstract}

\section{INTRODUCTION}

The study of $2 \mathrm{D}$ shapes and their similarities is a central problem in the field of vision. It arises in particular from the task of classifying and recognizing objects from their observed silhouette. Defining natural distances between 2D shapes creates a metric space of shapes, whose mathematical structure is inherently relevant to the classification task. Sharon and Mumford ${ }^{1}$ propose a method for the representation and comparison of $2 \mathrm{D}$ shapes based on constructions from the theory of conformal mapping. This intriguing metric space comes from using conformal mappings of $2 \mathrm{D}$ shapes into each other, via the theory of Teichmller spaces. In this space every simple closed curve in the plane (a "shape") is represented by a fingerprint which is a diffeomorphism of the unit circle to itself (a differentiable and invertible, periodic function). More precisely, every shape defines to a unique equivalence class of such diffeomorphisms up to right multiplication by a Mbius map. The fingerprint does not change if the shape is varied by translations and scaling and any such equivalence class comes from some shape. This coset space, equipped with the infinitesimal Weil-Petersson (WP) Riemannian norm is a metric space. In this space, the shortest path between each two shapes is unique, and is given by a geodesic connecting them. Their distance from each other is given by integrating the WP-norm along that geodesic. In this manuscript we provide an introduction to the basic fingerprint construction used in this space, and then examine how the geometry of a $2 \mathrm{D}$ shape is encoded in the fingerprint.

\subsection{Background: Complex Analytic Functions and Conformal Mapping}

Let $\Omega$ be a region in the complex plane $\mathbb{C}$. A function $f: \Omega \rightarrow \mathbb{C}$ is said to be complex analytic in $\Omega$ if it is differentiable at all $z_{0} \in \Omega$, that is

$$
f(z)=a_{0}+a_{1}\left(z-z_{0}\right)+o\left(\left|z-z_{0}\right|^{2}\right)
$$

If we consider $f^{\prime}\left(z_{0}\right)=a_{1}=r e^{i \theta}$ then we can interpret the complex derivative as an infinitesimal rotation and expansion. If we consider $\mathbb{C} \simeq \mathbb{R}^{2}$ and think $f: \mathbb{R}^{2} \rightarrow \mathbb{R}^{2}$ then the Jacobian of $f$ diagonalizes to a constant multiple of the identity. From either point of view, this gives us the 'angle preserving' property of complex analytic functions and is the connection to conformal geometry.

It is a remarkable fact that the existence of this complex derivative implies the existence of a convergent power series on all discs $D_{z_{0}} \subset \Omega$

$$
f(z)=\sum_{n=0}^{\infty} a_{n}\left(z-z_{0}\right)^{n}
$$

This power series is an essential tool when working with analytic functions.

We can also consider the 'extended complex plane' $\hat{\mathbb{C}}=\mathbb{C} \cup\{\infty\}$. Geometrically $\hat{\mathbb{C}}$ is the Riemann sphere: we identify points in the complex plane $\mathbb{C}$ with points on the unit sphere via stereographic projection (which is 
itself a conformal map of the plane to the sphere). We can speak of functions 'analytic at infinity' by considering power series in the variable $1 / z$. A function is analytic at infinity if it has a convergent power series for $|z|$ sufficiently large, i.e. there is some $M>0$ where the series

$$
f(z)=\sum_{n=0}^{\infty} a_{n}\left(\frac{1}{z}\right)^{n}
$$

converges for $|z|>M$ and in particular it converges to a finite value at infinity (i.e. $f(\infty)=a_{0}$ ). We can also make sense of 'convergence to infinity' by defining the topology on $\hat{\mathbb{C}}$ using the topology of the Riemann sphere: a sequence $\left\{z_{k}\right\}$ converges to infinity if $\left\{1 / z_{k}\right\} \rightarrow 0$.

Let $\Omega$ be a simply-connected region of the plane which omits at least one point. The Riemann Mapping Theorem then guarantees that we can always find a conformal map (a one-to-one complex analytic function) from the unit disc $\mathbb{D}$ to $\Omega$. This theorem underlies the conformal approach to shape representation.

Theorem 1.1 (Riemann Mapping Theorem). Let $\Omega$ be a simply-connected region other than the whole plane. Choose $w_{0} \in \Omega$. There exists an analytic function $f(z): \mathbb{D} \rightarrow \Omega$ which defines a one-to-one mapping of $\mathbb{D}$ onto $\Omega$. If we require $f(0)=w_{0}$ and $f i x \arg f^{\prime}(0)$ then this map is unique.

We will not give a proof of this theorem here. However, we will mention several constructive methods of computing the mapping $f$. We should first say a few words about the non-uniqueness of the Riemann map. There is a 3 (real) parameter group of conformal self-maps of the unit-disc. This group is sometimes called the Möbius group, also denoted $P S L(2, \mathbb{R})$, and the maps themselves are referred to as Möbius maps. Any Möbius map $\phi: \mathbb{D} \rightarrow \mathbb{D}$ can be written down explicitly; two particularly nice formulas are:

$$
\begin{aligned}
\phi(z) & =\frac{a z+b}{\bar{b} z+\bar{a}} \\
\phi(z) & =e^{i \theta} \frac{z-c}{1-\bar{c} z}
\end{aligned}
$$

The second formula provides a nice geometric interpretation of the three degrees of freedom. The first two degrees of freedom are the $x$ and $y$ coordinates of $c$, the image of 0 . The third degree of freedom is a rotation $\theta$.

Finally, we see that the Riemann map is unique only up to the Möbius group. That is, if $f: \mathbb{D} \rightarrow \Omega$ is a conformal map, then $f \circ \phi: \mathbb{D} \rightarrow \Omega$ is also a conformal map for any Möbius map $\phi$. The non-uniquess in the Riemann Mapping Theorem corresponds exactly to the Möbius group; intuitively we have the freedom to move $f(0)$ around the interior of our region $\Omega$ and to rotate the boundary values around $\partial \Omega$.

\subsection{Computing the Riemann Mapping}

We are interested in computing the Riemann map $f: \mathbb{D} \rightarrow \Omega$. While there are some elementary cases in which we can find an explicit formula for $f$, it is not possible to do so in general. There are a number of high-quality software packages available for numerical computation of the Riemann map; we mention a few here.

\subsubsection{Schwarz-Christoffel mapping}

Let $\Omega \subset \mathbb{C}$ be a polygon with vertices $\left\{v_{k}\right\}_{1}^{N}$ and interior angles $\left\{\pi \alpha_{k}\right\}_{1}^{N}$. It can be shown that if $f: \mathbb{D} \rightarrow \Omega$ is a conformal map, then it can be written as a Schwarz-Christoffel integral:

$$
f(z)=A+C \int^{z} \prod_{k=1}^{N}\left(z-u_{k}\right)^{\alpha_{k}-1}
$$

where the set of 'prevertices' $\left\{u_{k}\right\}$ are the preimages of the vertices, i.e. $\left\{u_{k}\right\}=\left\{f^{-1}\left(v_{k}\right)\right\}$. The constants $A$ and choice of basepoint for integration amount to translations of the resulting polygon; the constant $C$ allows for scale and rotation. Thus the computation of $f$ amounts to determining the locations of the prevertices $\left\{u_{k}\right\}$. We won't discuss this further here, but this is known as the 'Schwarz-Christoffel parameter problem' and its numerical solution has been implemented in several packages including the Matlab 'sc' toolbox by Tobin Driscoll. ${ }^{2-4}$ 


\subsubsection{Circle Packing}

Let $K$ be a simplicial 2-complex (think: a triangulation of an oriented surface). $K$ encodes the tangency relations of a set of circles.

A circle packing for $K$ is a configuration of circles s.t. for each vertex $v \in K$ there is a circle $c_{v}$, for each edge $<v, u>\in K$ the circles $c_{v}$ and $c_{u}$ are externally tangent and for each positively oriented face $\langle v, u, w>\in K$ the triple of tangent circles $\left\langle c_{u}, c_{v}, c_{w}>\right.$ is positively oriented.

Theorem 1.2 (Circle Packing Theorem). Let $K$ be a closed disc. There exists a circle packing $P_{K}$ for $K$ in $\mathbb{D}$ that is univalent, unique up to Möbius transformations of $\mathbb{D}$ and whose boundary circles are horocycles.

There is a simple algorithm for computing these packings, and remarkably the packings converge under refinement to a complex analytic map: if one uses increasingly fine hexagonal packings in some domain $\Omega$, the packing of the disc with the same tangency relations will converge to produce the Riemann map. This method has been implemented by Ken Stephenson..$^{5,6}$

\subsubsection{Zipper}

The final algorithm we'll mention is called 'Zipper', due to Don Marshall. ${ }^{7}$ It finds the conformal map from the half-plane to a region $\Omega$. The map is represented as a composition of elementary linear fractional maps; each map in the composition is a map to a slit half-plane. The method can be viewed as a discrete solution to the Loewner equation and avoids many numerical issues which arise in other methods.

\subsection{Physics of the Riemann Map}

We claim that the boundary derivatives of conformal maps encode useful features of the geometry. This can be made intuitive by appealing to the physics of conformal mapping. There is a close connection between conformal mapping and the physics of heat flow and electrostatics. In particular, conformal maps can be used to construct Green's functions for the Laplace operator on simply-connected domains.

Our first step is to observe that harmonic functions are preserved under conformal maps. That is, let $\Omega \subset \mathbb{C}$ be a domain and let $f$ map $\mathbb{D}$ conformally onto $\Omega$. Suppose further that $h: \mathbb{D} \rightarrow \mathbb{R}$ satisfies $\triangle h=0$ in $\mathbb{D}$. Then by explicit calculation and application of the Cauchy-Riemann conditions one easily shows that $\triangle\left(h \circ f^{-1}\right)=0$ in $\Omega$.

We can use this preservation of harmonicity to construct potential functions in an arbitrary simply-connected planar domain $\Omega$. We'll start by explicitly identifying the Green's function for a simple domain: the unit disc. Let $g: \mathbb{D} \rightarrow \mathbb{R}$ be Green's function for the Laplace operator in the unit disc with pole at 0 . By definition $g$ is harmonic in the disc with a logarithmic pole at the origin; explicitly $g=\log \frac{1}{r}$. Physically, $g$ has the following significance in terms of heat flow or electrostatics:

1. The steady-state temperature distribution in the unit disc resulting from a heat source placed at the origin when the boundary of the disc is fixed at zero degrees.

2. The electrical potential in the unit disc resulting from a unit point charge at the origin with the unit circle connected to ground.

Potential theory further tells us that the stream lines of the flow can be found by following the gradient of the potential function and the equipotential lines are the level sets of the potential function; the equipotentials are orthogonal to the stream lines. In the case of the disc with a pole at the origin, the stream lines are the radii and the equipotentials are concentric circles about the origin. If we take $\Omega$ to be some simply-connected planar domain, we may appeal to the Riemann Mapping Theorem for help understanding basic electrostatics or heat flow. If $f: \mathbb{D} \rightarrow \Omega$ is a Riemann map, then (by our observation above) $g \circ f^{-1}$ is harmonic in $\Omega$ except for a logarithmic pole at $f(0)$. Thus $g \circ f^{-1}$ is the potential function we seek; it describes the steady-state heat distribution or electrostatic potential in $\Omega . f$, the conformal map, maps the stream lines and equipotentials on the disc to stream lines and equipotentials on $\Omega$. 
Conversely, we may gain intuition about the Riemann map and its derivatives through our intuitive understanding of electricity or heat. This is what we wish to do here. Consider the heat flowing across the boundary of some domain $\Omega$ in the heat-flow model. Intuition tells us that the heat flow across the boundary will be greater at boundary points near the source and lesser at points which are far away from the source. From physics we know that the heat flux on the boundary is given by $\frac{\partial g}{\partial \mathbf{n}}$, the derivative of the Green's function in the normal direction $\mathbf{n}$. Since $\frac{\partial g}{\partial \mathbf{n}}=\left|\frac{1}{f^{\prime}}\right|$ along the boundary of $\Omega$, we deduce that $\left|f^{\prime}\right|$ is large when heat flow is small and $\left|f^{\prime}\right|$ is small where heat flow is large. See figure 1.
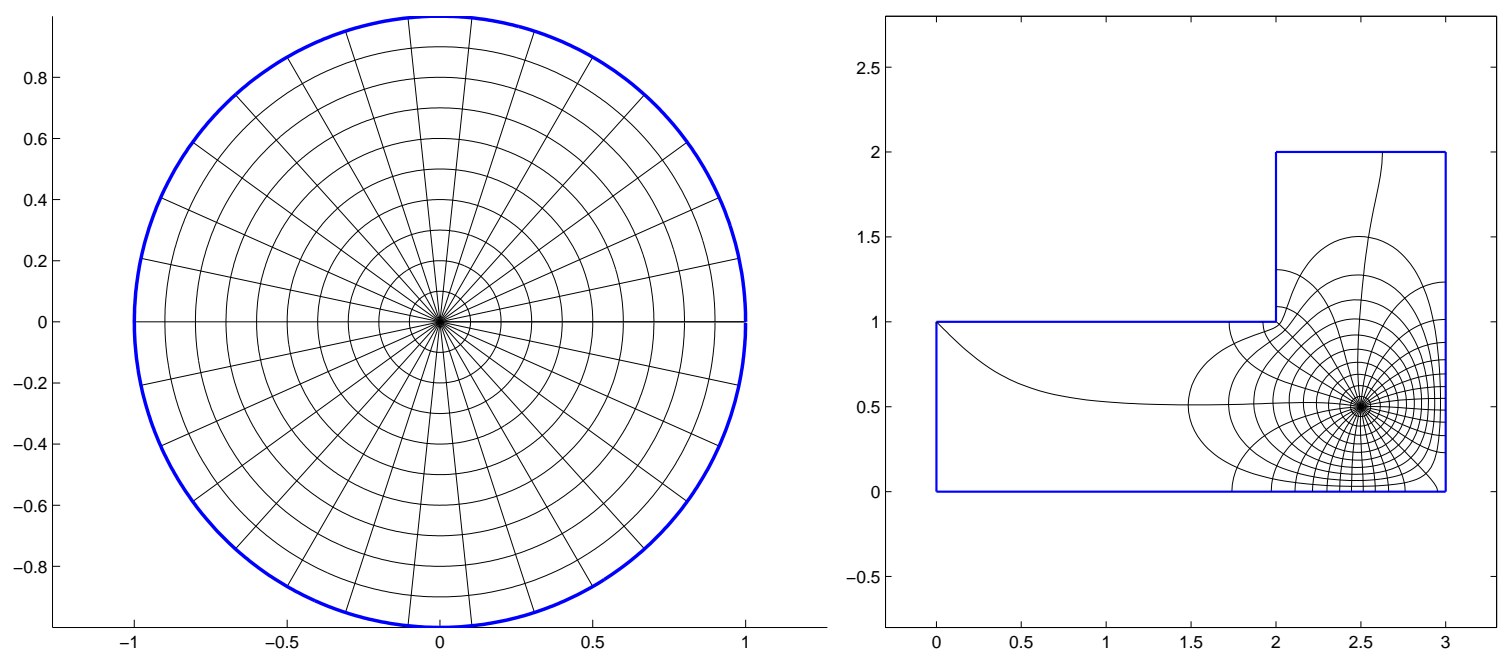

Figure 1. Streamlines and equipotentials on the disc (left) are mapped to streamlines and equipotentials for an L-shaped region by conformal maps. Note that streamlines cluster at nearby points on the boundary and concave corners while avoiding convex corners.

\section{FINGERPRINTS AND CONFORMAL WELDING}

We need to introduce some notations. Let $\Gamma \subset \mathbb{C}$ be a simple closed curve. Then we write:

$\Delta_{-}:$the interior of the unit disc

$\Delta_{+}$: the exterior of the unit disc

$\Gamma_{-}$: the union of $\Gamma$ and the region it encloses

$\Gamma_{+}$: the union of $\Gamma$ and the region outside $\Gamma$

$\Phi_{-}:$the Riemann map $\Delta_{-} \rightarrow \Gamma_{-}$

$\Phi_{+}$: the Riemann map $\Delta_{+} \rightarrow \Gamma_{+}$

The existence of $\Phi_{-}$is guaranteed by the Riemann Mapping theorem, and $\Phi_{-}$is unique up to Möbius transformations of the disc. To see that the Riemann mapping theorem guarantees the existence of the exterior map $\Phi_{+}$, observe that we can write $\Phi_{+}$in the form

$$
\Phi_{+}(z)=\frac{1}{f(1 / z)}
$$

where $f$ is some map from the interior of the disc to the complex inversion of $\Gamma_{+}$, i.e. the set $\left\{z \in \mathbb{C}: 1 / z \in \Gamma_{+}\right\}$. The existence of such $f$ is guaranteed by the Riemann mapping theorem. So $\Phi_{+}$exists. However, we remove the ambiguity in the construction of $\Phi_{+}$by requiring that $\Phi_{+}(\infty)=\infty$ and that $\Phi_{+}$takes the tangent at infinity to the positive real axis. So $\Phi_{+}$is unique.

Now we define the 'fingerprint' of $\Gamma$ on the unit circle $S^{1}$

$$
\Psi=\Phi_{+}^{-1} \circ \Phi_{-}
$$


$\Psi$ is a diffeomorphism of $S^{1}$ to itself and is unique up to the Möbius ambiguity remaining in $\Phi_{-}$. That is, if we denote the space of simple closed curves by $\mathcal{S}$ then via $\Psi$ we have the 'fingerprint' map

$$
\mathcal{S} \rightarrow \operatorname{Diff}\left(\mathrm{S}^{1}\right) / P S L(2, \mathbb{R})
$$

This map is a bijection up to translations and scalings of $\Gamma$. Some examples are shown in the next section; see figures 2,3 , and 4 .

\subsection{Examples: The Fingerprint Encodes the Geometry}

Figures 2, 3, and 4 demonstrate some shapes and their conformal fingerprints. It is apparent from these figures that the fingerprint $\Psi$ encodes information about the domain in the derivative $\Psi^{\prime}$. The derivative $\Psi^{\prime}$ can be seen to be influenced by two factors: the boundary curvature near a point of interest and the distance, in some sense, between $\Phi_{-}(0)$ and the boundary point of interest. That is,

- $\Psi^{\prime}$ is largest at points of convexity (positive curvature) which are far from $\Phi_{-}(0)$

- $\Psi^{\prime}$ is smallest at points of concavity (negative curvature) which are neat $\Phi_{-}(0)$

When we say a boundary point is 'near' or 'far' from $\Phi_{-}(0)$ we take our distances along paths entirely contained inside the domain.

These results simply reflect the behavior of the boundary derivatives, since $\Psi=\Phi_{+}^{-1} \circ \Phi_{-}$.
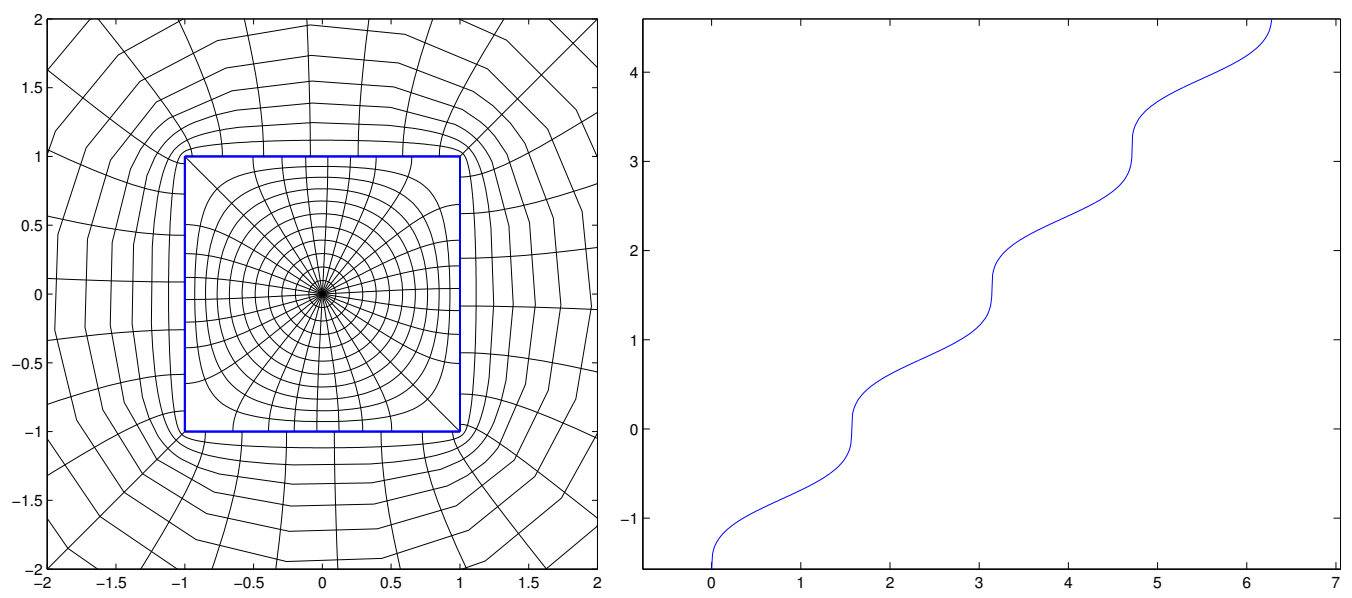

Figure 2. The fingerprint of a square. On the left we see the images of circles and radial lines under the interior map $\Phi_{-}$ and exterior map $\Phi_{+}$. On the right is the fingerprint $\Psi=\left.\Phi_{+}^{-1} \circ \Phi_{-}\right|_{S^{1}}$. Large values of $\Psi^{\prime}$ correspond to corners while moderate values correspond to the sides of the square.

\subsection{Conformal Axes}

The boundary derivatives of the interior map and the derivatives of the fingerprint can both be used to construct a sort of axis on the interior of the shape. These constructions rely on the behavior of the boundary derivatives of conformal maps.

Definition 2.1 (Interior Conformal Axis). Let $\Omega$ be a domain in $\mathbb{C}$ and let $f: \mathbb{D} \rightarrow \Omega$ conformal with $f(0)=w$ for some $w \in \Omega$. The interior conformal axis of $\Omega$ is the set of points $w \in \Omega$ where the boundary derivative function $\left|f^{\prime}\left(e^{i \theta}\right)\right|$ has multiple equal minima when $f(0)=w$.

This construction can be viewed as a conformally-natural version of the medial axis of a shape. The medial axis is defined as the set of centers of all inscribed circles with at least two points of tangency with the boundary $\partial \Omega$. In the conformal setting, for any point $e^{i \theta}$, the boundary derivative $\left|f^{\prime}\left(e^{i \theta}\right)\right|$ will decrease as $f(0)$ is moved 

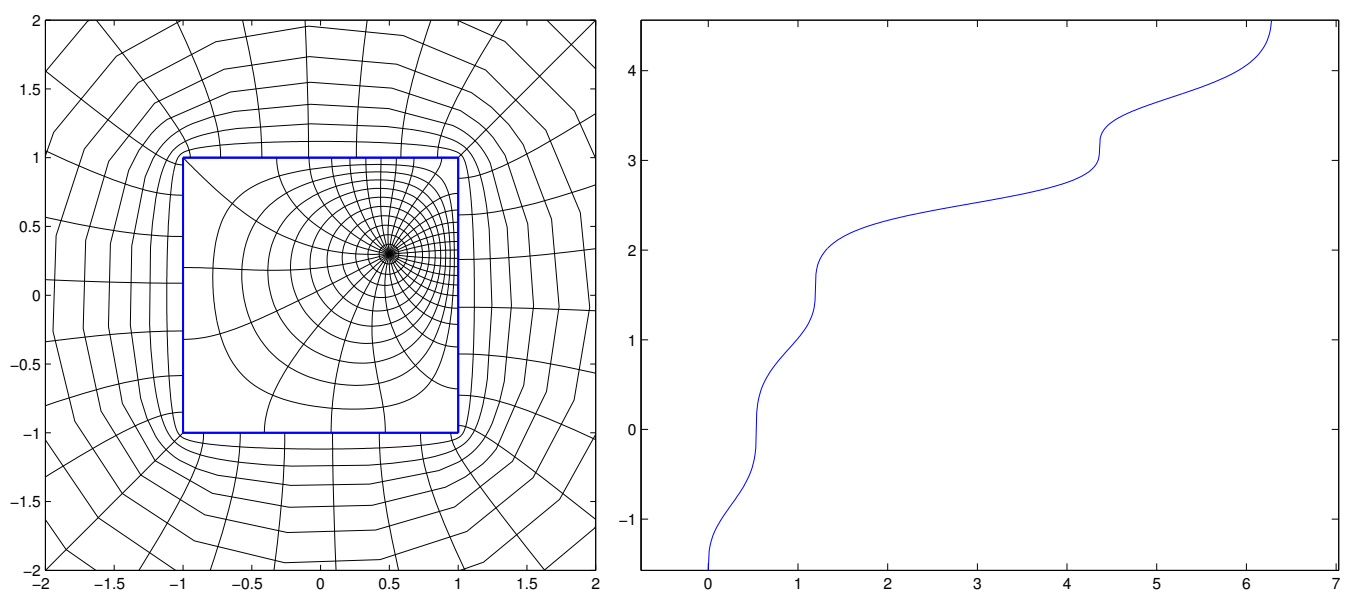

Figure 3. The fingerprint is unique only up to Möbius maps of the interior map. If we alter $\Phi_{-}(0)$ we can look at different representatives of the equivalence classes. The essential character is preserved, but the derivative $\Psi^{\prime}$ becomes much smaller along boundary segments near $\Phi_{-}(0)$.
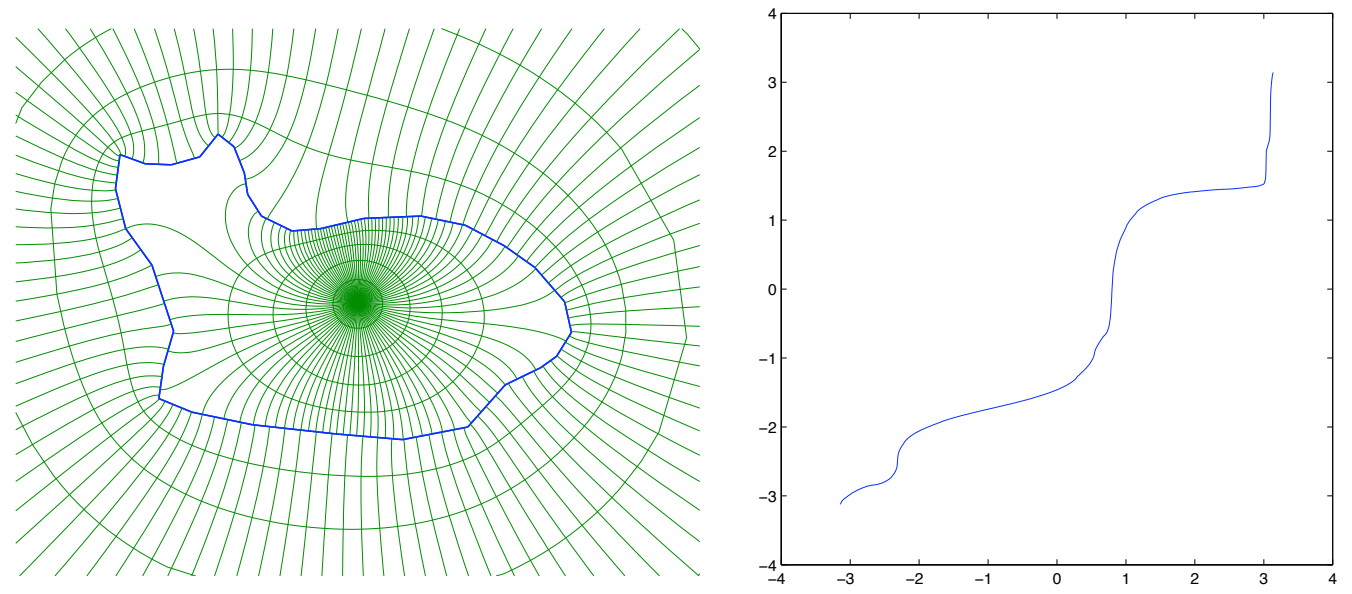

Figure 4. The fingerprint of a cat. Large positive boundary curvature corresponds to large $\Psi^{\prime}$ and negative curvature corresponds to small $\Psi^{\prime}$. 
toward $f\left(e^{i \theta}\right)$. Thus this interior conformal axis tends to include points in $\Omega$ which are roughly equidistant from two points on the boundary $\partial \Omega$.

The fingerprint yields a similarly-natural construction:

Definition 2.2 (Conformal Axis). Let $\Omega$ be a domain in $\mathbb{C}$ with fingerprint $\Psi$ relative to some basepoint $w \in \Omega$. The conformal axis of $\Omega$ is the set of points $w$ where $\Psi^{\prime}$ has multiple equal minima.

The conformal axis can also be understood as a conformally-natural analogue of the medial axis. The derivative of the fingerprint $\Psi^{\prime}=\left|\Phi_{+}^{\prime}\right|\left|\Phi_{-}^{\prime}\right|$ is completely determined by the derivatives of the interior and exterior maps, and the same qualitative behavior influences the point set making up the axis.

\subsection{Conformal Welding}

While we have shown how to produce $\Psi$ given $\Phi_{-}, \Phi_{+}$, we have not shown that we can go the other way and produce a $2 \mathrm{D}$ shape given a fingerprint. That is, we still must show how to obtain $\Gamma, \Phi_{-}$, and $\Phi_{+}$given some representative $\Psi$ from an equivalence class in $\operatorname{Diff}\left(\mathrm{S}^{1}\right) / P S L(2, \mathbb{R})$. There turns out to be a very practical and effective way of performing this computation.

Abstractly, we construct a Riemann surface $X$ by identifying the boundaries of $\Delta_{-}$and $\Delta_{+}$according to $\Psi$. This is called welding and yields a topological sphere. However, we can apply the result that there exists a conformal map $\Phi: X \rightarrow \hat{\mathbb{C}}$, and so we have that $\left.\Phi\right|_{\Delta_{-}}=\Phi_{-}$and $\left.\Phi\right|_{\Delta_{+}}=\Phi_{+}$. Then $\Gamma$ is the image of the unit circle, i.e. $\partial \Delta_{-}$or $\partial \Delta_{+}$, under $\Phi$. This is enough to show existence of welds.

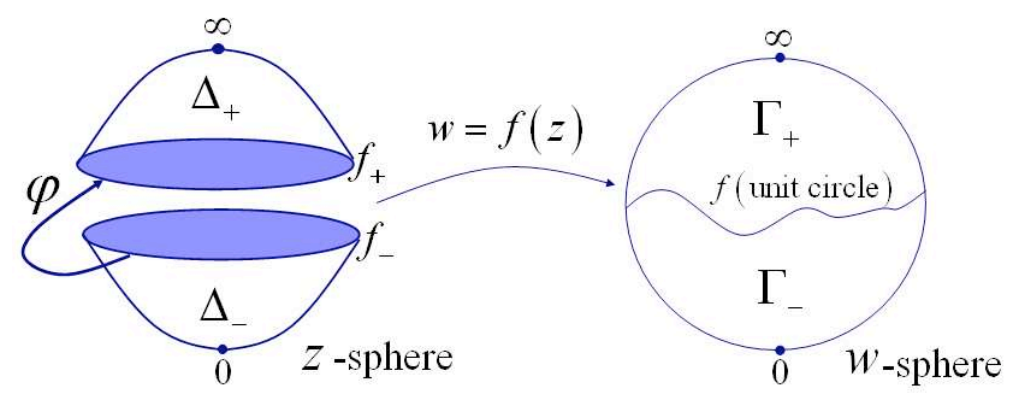

We'll discuss an algorithm for solving the welding problem based on the Hilbert transform. Let $k(x)=1 / x$ and then on the real line we can define the Hilbert transform

$$
H f(x)=(f * k)(x)=\int f(y) k(x-y) \mathrm{d} y
$$

Note that if we interpret the integral in the sense of principal values at the kernel's singularity then it is wellbehaved as long as $f$ is continuous.

Since the derivative of the function $\operatorname{sign}(x)$ is $2 \delta_{0}$, we compute the Fourier transform

$$
\begin{aligned}
\widehat{\left(\operatorname{sign}^{\prime}\right)}(\xi) & =2 \widehat{\delta_{0}} \\
(2 \pi i \xi) \widehat{\operatorname{sign}}(\xi) & =2 \\
\pi \widehat{\operatorname{sign}}(\xi) & =\frac{2}{\xi} \\
\Longrightarrow\left(\frac{1}{x}\right) & =-\pi i \operatorname{sign}(\xi)
\end{aligned}
$$

Therefore,

$$
\widehat{H f}(\xi)=\hat{f} \hat{k}=-\pi i \operatorname{sign}(\xi) \hat{f}(\xi)
$$


For periodic functions defined on the unit circle the equivalent operation is convolution with $\cot (\theta / 2)$, or multiplication of the Fourier coefficients by $-i \operatorname{sign}(n)$ and this is the case we find ourselves in.

Consider the function $\Phi_{+}$. It has a series expansion

$$
\Phi_{+}(z)=b z+a_{0}+a_{1} \frac{1}{z}+a_{2} \frac{1}{z^{2}}+\ldots, \quad b \in \mathbb{R}_{+}
$$

Normalize so $b=1$ since the construction is not sensitive to scale. On the unit circle we have $z=e^{i \theta}$ so the series for $\Phi_{+}$becomes

$$
\Phi_{+}\left(e^{i \theta}\right)=b e^{i \theta}+a_{0}+a_{1} e^{-i \theta}+a_{2} e^{-2 i \theta}+\ldots=e^{i \theta}+\sum_{n=0}^{\infty} a_{n} e^{-i n \theta}
$$

Let $F(\theta)=\Phi_{+}\left(e^{i \theta}\right)$ so we have

$$
\begin{aligned}
i H F(\theta) & =e^{i \theta}-\sum_{n=1}^{\infty} a_{n} e^{-i n \theta} \\
& =2 e^{i \theta}+a_{0}-F(\theta)
\end{aligned}
$$

This may be interpreted as the Hilbert-transformed version of the statement that $\Phi_{+}$is analytic outside the unit disc with a simple pole and derivative 1 at infinity.

$\Phi_{-}$, on the other hand, is analytic on the closed disc. If we expand it in power series we have

$$
\Phi_{-}(z)=c_{0}+c_{1} z+c_{2} z^{2}+\ldots
$$

Now since on the circle

$$
\Phi_{-}\left(e^{i \theta}\right)=(F \circ \Psi)(\theta)
$$

we obtain

$$
i H(F \circ \Psi)(\theta)=\sum_{c=1}^{\infty} c_{n} z^{n}=(F \circ \Psi)(\theta)-c_{0}
$$

This is interpreted as the Hilbert transform of the statement that $\Phi_{-}$is analytic on the disc. Now subtracting our two conditions we obtain

$$
i H(F \circ \Psi) \circ \Psi^{-1}-i H(F)=2 F-\left(a_{0}+c_{0}\right)-2 e^{i \theta}
$$

Since $F$ is defined only up to translation, we replace $F$ by $F-\frac{a_{0}-c_{0}}{2}$. Now define

$$
K(F)=\frac{i}{2}\left(H(F)-H(F \circ \Psi) \circ \Psi^{-1}\right)
$$

and we can rewrite the preceding expression as an integral equation

$$
K(F)+F=e^{i \theta}
$$

We can in fact show that $K$ is a smooth integral operator (and hence a compact operator). We first expand $K$ :

$$
\begin{aligned}
K(F)(\theta)= & \frac{i}{2} \int_{S^{1}} \cot \left(\frac{\theta-\phi_{1}}{2}\right) F\left(\phi_{1}\right) \mathrm{d} \phi_{1}-\cot \left(\frac{\Psi^{-1}(\theta)-\phi_{2}}{2}\right) F\left(\Psi\left(\phi_{2}\right)\right) \mathrm{d} \phi_{2} \\
& \text { Let } \phi_{3}=\Psi\left(\phi_{2}\right): \\
= & \frac{i}{2} \int_{S^{1}} \cot \left(\frac{\theta-\phi_{1}}{2}\right) F\left(\phi_{1}\right) \mathrm{d} \phi_{1}-\left(\Psi^{-1}\right)^{\prime}\left(\phi_{3}\right) \cot \left(\frac{\Psi^{-1}(\theta)-\Psi^{-1}\left(\phi_{3}\right)}{2}\right) F\left(\phi_{3}\right) \mathrm{d} \phi_{3} \\
& \text { Gathering terms yields } \\
= & \frac{i}{2} \int_{S^{1}}\left(\cot \left(\frac{\theta-\phi_{1}}{2}\right)-\left(\Psi^{-1}\right)^{\prime}\left(\phi_{1}\right) \cot \left(\frac{\Psi^{-1}(\theta)-\Psi^{-1}\left(\phi_{1}\right)}{2}\right)\right) F\left(\phi_{1}\right) \mathrm{d} \phi_{1}
\end{aligned}
$$


The simple poles will cancel and the operator $K$ is smooth. We now wish to solve the equation $(I+K)^{-1}\left(e^{i \theta}\right)=F$. The Fredholm alternative tells us that either $(I+K)$ is invertible or it has a kernel. If $(I+K)$ were to have a kernel, then any functions in it would define analytic functions on the entire topological sphere constructed via welding. To see this, one must trace through the algebra and see that the analog of equation 25 for a general $F$ is that $K F+F$ is equal to the sum of the singular parts of the extensions of $F$ to the interior and exterior of the disc. So $K F+F=0$ means that $F$ 's extensions have no singular parts at all; hence they form an analytic function on the sphere which must reduce to a constant by Liouville's theorem. Therefore the kernel would consist of constants. But $K$ kills constants, so they are not in the kernel and we may solve the integral equation to obtain the welding.

\section{SUMMARY}

We introduce a representation for 2D shapes, the conformal "fingerprint", which is based on conformal mappings. This fingerprint encodes the Euclidean geometry of a planar shape in a natural way; in particular it encodes the Euclidean curvature of the boundary and contains a conformally-natural analogue of the medial axis of a planar

domain. Intuitively, many of these connections can be understood by appealing to potential theory: classical electrostatics and heat flow both have close relationships to conformal mapping. We feel that this conformal approach may be viewed as a continuous analogue of axis-based approaches to $2 \mathrm{D}$ shape, e.g. Kimia et al, ${ }^{8}$ and we expect that the axis-type constructions will play an important role in understanding geodesics in this metric space of shapes.

\section{REFERENCES}

1. E. Sharon and D. Mumford, "2d-shape analysis using conformal mapping," Int. J. Comput. Vision 70(1), pp. 55-75, 2006.

2. T. A. Driscoll and S. A. Vavasis, "Numerical conformal mapping using cross-ratios and delaunay triangulation," SIAM Sci. Comput. 19, pp. 1783-1803, 1998.

3. T. Driscoll, "A matlab toolbox for schwarz-christoffel mapping," ACM Trans. Math. Soft. 22, pp. 168-186, 1996.

4. T. Driscoll and L. Trefethen, Schwarz-Chrsitoffel Mapping, Cambridge University Press, 2002.

5. C. R. Collins and K. Stephenson, "A circle packing algorithm," Computational Geometry: Theory and Applications 25, pp. 233-256, 2003.

6. K. Stephenson, Introduction to Circle Packing, Cambridge University Press, 2005.

7. D. Marshall and S. Rohde, "Convergence of the zipper algorithm for conformal mapping," ArXiv Mathematics e-prints, May 2006.

8. T. B. Sebastian, P. N. Klein, and B. B. Kimia, "Recognition of shapes by editing shock graphs," ICCV , pp. 755-762, 2001. 